\title{
Expression and clinical value of SALL4 in renal cell carcinomas
}

\author{
${\text { JIANPING } \mathrm{CHE}^{1 *}, \text { PENGFEI WU }}^{2 *}$, GUANGCHUN WANG $^{2}, \mathrm{XUDONG} \mathrm{YAO}^{2}$, \\ JUNHUA ZHENG $^{1}$ and CHANGCHENG GUO ${ }^{2}$ \\ ${ }^{1}$ Department of Urology, The Affiliated Shanghai Tenth People's Hospital, Nanjing Medical University; \\ ${ }^{2}$ Department of Urology, Shanghai Tenth People's Hospital, Tongji University, Shanghai 200072, P.R. China
}

Received July 22, 2019; Accepted March 30, 2020

DOI: $10.3892 / \mathrm{mmr} .2020 .11170$

\begin{abstract}
The aim of the present study was to investigate the expression of spalt like transcription factor 4 (SALL4) in the three most common types of renal cell carcinomas (RCC) [clear cell RCC (ccRCC), papillary renal cell carcinoma (pRCC) and chromophobe RCC (chRCC)], and the association with the overall survival (OS) of patients. The Cancer Genome Atlas (TCGA) database and RCC samples were used to investigate the expression levels of the SALL4 gene and its association with the OS in the three types of RCC based on the analysis of the transcriptome, copy number and survival data. It was found that SALL4 was highly expressed in ccRCC and pRCC tumor tissue, and low mRNA expression level of SALL4 indicated a prolonged survival in both ccRCC and pRCC. This mRNA expression level was associated with pathological Tumor-Node-Metastasis stage, $\mathrm{M}$ and $\mathrm{T}$ stages in both ccRCC and pRCC. The analysis of the enriched pathway results suggested that SALL4 may act via translation initiation, and that the related genes promoted the progression of RCC. Moreover, the high expression level of SALL4 was detected in RCC samples and serum from patients. It was demonstrated that SALL4 promotes increased viability in RCC cells. Therefore, the present results suggest that SALL4 may be a sensitive and specific cancer biomarker in ccRCC and pRCC. Furthermore, targeting of SALL4 may improve RCC therapy and prolong the survival of patients with ccRCC or pRCC.
\end{abstract}

Correspondence to: Dr Junhua Zheng, Department of Urology, The Affiliated Shanghai Tenth People's Hospital, Nanjing Medical University, 301 Yanchang Road, Shanghai 200072, P.R. China

E-mail: zhengjh0471@sina.com

Dr Changcheng Guo, Department of Urology, Shanghai Tenth People's Hospital, Tongji University, 301 Yanchang Road, Shanghai 200072, P.R. China

E-mail: greatwall063030@126.com

*Contributed equally

Key words: renal cell carcinomas, spalt like transcription factor 4, SALL4, The Cancer Genome Atlas, survival, clinical value

\section{Introduction}

Renal cell carcinoma (RCC) is the ninth most common malignant tumor, accounting for $2-3 \%$ of all adult malignancies (1). In the USA, RCC is the sixth leading cause of cancer-related deaths in men, while the eighth leading cause in women (2). Currently, the worldwide incidence rate of malignancy increases by $\sim 2 \%$ every year (3). In 2012, 84,400 new RCC cases were diagnosed, of which 34,700 resulted in kidney cancer-related deaths in the European Union (3). Surgery is still the optimal treatment for primary RCC, while for advanced metastatic RCC several targeted therapies have been approved by the Food and Drug Administration (FDA), including sunitinib (4). Although these FDA-approved therapies have extended the survival time of patients with advanced RCC, the response rate of targeted therapy is weak and the 5-year survival rate is $<10 \%$ (5). Therefore, studies have focused on novel, efficacious strategies for the treatment of metastatic RCC.

The spalt-like gene family consists of four members, including spalt like transcription factor (SALL) 1, SALL2, SALL3 and SALL4 (6). SALL4 was cloned based on the DNA sequence homology to the homeotic gene in Drosophila, spalt $(7,8)$. SALL4 is enriched in embryonic cells and plays a major role in self-renewal capability, while its expression is silenced in mature adults $(9,10)$. However, SALL4 can also be re-expressed in various cancer types (11-16); it was first recognized as an oncogene in leukemia (17). Previous studies have shown that SALL4 is overexpressed in various tumors, and may play a role in tumorigenesis and tumor progression (18). Furthermore, SALL4 may have a function in different subclasses of hepatocellular carcinoma (HCC), and is a key factor in maintaining the properties of cancer stem cells $(19,20)$. Therefore, targeting the SALL4 gene as a potential therapeutic strategy has been demonstrated in various cancer types. In acute myeloid leukemia and HCC, a peptide that can compete with SALL4 to interact with the HDAC complex has been used to treat patients $(21,22)$.

However, the expression level and function of SALL4 in different subtypes of RCC are not fully understood. The present study aimed to investigate the expression level and function of SALL4 using the data from The Cancer Gene Atlas (TCGA) to understand the molecular mechanisms underlying SALL4 expression. The present study also assessed the function of SALL4 in different types of RCC to ascertain whether it has vital clinical implications. If SALL4 promotes RCC 
malignancy, then therapeutic strategies targeting SALL4 using PTEN (23) or entinostat (24) may have clinical therapeutic efficacy.

\section{Materials and methods}

TCGA. The RNA-seq data from the cohorts of 604 clear cell RCC (ccRCC), 320 papillary RCC (pRCC) and 89 chromophobe RCC (chRCC) cases were extracted from TCGA database (https://xena.ucsc.edu/). In addition, the clinical outcomes including the pathological Tumor-Node-Metastasis (TNM) stage (25), $\mathrm{T}$ and $\mathrm{M}$ stages and the overall survival (OS) were assessed using the Xena platform (http://xena. ucsc.edu/). These three cohorts included $\sim 20,500$ gene data points. In addition, clinical information, including the time to last follow-up, survival state and sex of each patient from the TCGA database was extracted. In addition to SALL4 gene data, copy number data and the clinical relevance were retrieved from TCGA. The SALL4 expression level between tumor and normal tissues in different tumors was analyzed using FireBrowse software (Broad Institute GDAC Firebrowse version 1.1.35; http://firebrowse.org/).

Association between SALL4 gene and survival. The three types of patients with RCC were divided into two groups based on the level of SALL4 mRNA expression (high expression SALL4 or low expression SALL4 group) or copy number (SALL4 high copy number or SALL4 low copy number group). Kaplan-Meier analysis was performed using GraphPad Prism 7 (GraphPad Software, Inc.) or Xena (http://xena.ucsc.edu/).

SALL4-associated gene expression and enriched pathway analysis. In total, the gene expression of 10 patients from TCGA databse with high expression of SALL4 and 10 with low expression from the TCGA database were analyzed. These data were obtained using the $\mathrm{WebMeV}$ cloud platform for analyzing and visualizing cancer genomic data (http://mev. tm4.org/\#/datasets/tcga) using the voom function.

SALL4 expression and its function in RCC cells and samples. Between September 2018 and June 2019, 10 patients with RCC and 10 healthy control patients were enrolled in the present study at the Department of Shanghai Tenth People's Hospital, Tongji University. Preoperative clinical data for each patient, including complete blood count, were entered into a computerized database. Then, two different types of tissues from each patient with RCC, including RCC tumor tissue and another tumor-free sample was taken at $>2 \mathrm{~cm}$ from the tumor edge following surgical resection. These specimens were preserved in $10 \%$ formaldehyde solution at $62^{\circ} \mathrm{C}$ for $1 \mathrm{~h}$ and embedded in paraffin. The detail information of the patients is documented in Table I. The current study was performed according to the protocol approved by the Ethics Committee of Shanghai Tenth People's Hospital, Tongji University School of Medicine. Written informed consent for participation was obtained from each patient.

Cell culture. OSRC-2,HK2, ACHN, 293T and SW839 cell lines were purchased from the American Type Culture Collection. All cell lines were cultured in DMEM (Invitrogen; Thermo Fisher Scientific, Inc.) supplemented with 10\% FBS (Thermo
Fisher Scientific, Inc.), penicillin $(25 \mathrm{U} / \mathrm{ml})$, streptomycin $(25 \mathrm{~g} / \mathrm{ml})$ and $1 \% \mathrm{~L}$-glutamine. All cell lines were cultured in a $5 \% \mathrm{CO}_{2}$ humidified incubator at $37^{\circ} \mathrm{C}$.

Lentivirus packaging. The pLKO-sh-SALL4, the psAX2 packaging plasmid and pMD2G envelope plasmid from George Whipple lab of University of Rochester were transfected into 293T cells using the standard calcium chloride transfection method (26) for $48 \mathrm{~h}$ to get the lentivirus soup. The lentivirus soup was collected and concentrated by density gradient centrifugation at $4,000 \mathrm{x}$ g for $20 \mathrm{~min}$ at $4^{\circ} \mathrm{C}$, and then frozen at $-80^{\circ} \mathrm{C}$. The cells were transfected using Lipofectamine ${ }^{\circledR}$ 2000 (Invitrogen; Thermo Fisher Scientific, Inc.) The standard transfection method of the SW839 and OSRC-2 cells with sh-SALL4 was as follows: $4 \mu \mathrm{l}$ aqueous solution containing $4 \mu \mathrm{g}$ of DNA was mixed with $10 \mu 12.5 \mathrm{M} \mathrm{CaCl}_{2}$ solution. The dispersion was incubated for $5 \mathrm{~min}$. The volume of the dispersion was adjusted to $100 \mu \mathrm{l}$ with water and $100 \mu \mathrm{l}$ HEPES buffered saline solution $(280 \mathrm{mM} \mathrm{NaCl}, 10 \mathrm{mM} \mathrm{KCl}, 1.5 \mathrm{mM}$ $\mathrm{Na}_{2} \mathrm{HPO}_{4}, 12 \mathrm{mM}$ dextrose, $50 \mathrm{mM}$ HEPES, $\mathrm{pH} 7.05 \pm 0.01$ ) was added. Cell culture medium (RPMI 1640 with $15 \%$ fetal calf serum; Thermo Fisher Scientific, Inc.) was added up to $1 \mathrm{ml}$. The culture medium was removed was removed from the cells, and the transfection mixture was added. After 7-h incubation at $37^{\circ} \mathrm{C}$, the transfection mixture was replaced by fresh cell culture medium. The sequences of sh-SALL4 were as follows: Forward, 5'-CGCGTCCAGAGAATCCCTGTGACTTTACGG ACCCGGTCGACGTCCGTAAAGTCACAGGGATTCTCT GGCATTTTTG-3' and reverse, 5'-CGCAAAAACCAGAGA ATCCCTGTGACTTTACGGACGTCGACCGGGTCCGTA AAGTCACAGGGATTCTCTGGCATATCTA-3'.

Immunohistochemistry (IHC). Human RCC sections (thickness, $5 \mu \mathrm{m})$ were deparaffinized in a xylene solution $(100 \%)$ and rehydrated using gradient ethanol concentrations ( $70 \%$ for $5 \mathrm{~min}, 80 \%$ for $5 \mathrm{~min}, 90 \%$ for $5 \mathrm{~min}$ and $100 \%$ for $5 \mathrm{~min}$ ). Endogenous peroxidase activity was blocked with $3 \%$ hydrogen peroxide for $10 \mathrm{~min}$ at $37^{\circ} \mathrm{C}$. Heat-induced antigen retrieval was performed for all sections with $0.01 \mathrm{M}$ sodium citrate (pH 6.0) at $98^{\circ} \mathrm{C}$ for $30 \mathrm{~min}$. Then, IHC staining was performed with specific primary antibodies against SALL 4 at $37^{\circ} \mathrm{C}$ for $120 \mathrm{~min}$ (cat. no. ab57577; dilution, 1:100; Abcam). The sections were subsequently incubated with a horseradish peroxidase-conjugated IgG H\&L secondary antibody (cat. no. ab205719; dilution, 1:1,000; Abcam) at $37^{\circ} \mathrm{C}$ for $60 \mathrm{~min}$. Staining was performed using diaminobenzidine for $5 \mathrm{~min}$ at room temperature followed by counterstaining with hematoxylin for $1 \mathrm{~min}$ at room temperature. The sections were dehydrated and fixed using a graded ethanol series (70\% for $5 \mathrm{~min}, 80 \%$ for $5 \mathrm{~min}$, $90 \%$ for $5 \mathrm{~min}$ and $100 \%$ for $5 \mathrm{~min}$ ), treated with xylene for 10 min at room temperature and mounted with Permount ${ }^{\mathrm{TM}}$ mounting medium (Thermo Fisher Scientific, Inc.) The slides were observed using a light microscope under five random high-power fields (magnification, $\mathrm{x} 400$ ).

Reverse transcription-quantitative PCR (RT-qPCR). For RNA extraction, total RNAs were isolated using TRIzol ${ }^{\circledR}$ reagent (Invitrogen; Thermo Fisher Scientific, Inc.). Then, $1 \mu \mathrm{g}$ of total RNA was subjected to RT using an RT-PCR kit (Takara Bio, Inc.) according to the manufacturer's instructions. qPCR was 
Table I. Patient data of the RCC cases $(\mathrm{N}=10)$ and the healthy controls $(\mathrm{N}=10)$.

\begin{tabular}{|c|c|c|c|c|c|c|c|c|c|}
\hline \multirow[b]{2}{*}{ Group } & \multirow[b]{2}{*}{ Sex } & \multirow[b]{2}{*}{ Patients } & \multirow[b]{2}{*}{ Mean age, years } & \multicolumn{3}{|c|}{ TNM stage } & \multicolumn{3}{|c|}{ Pathology } \\
\hline & & & & T1a & $\mathrm{T} 1 \mathrm{~b}$ & $\mathrm{~T} 2$ & ccRCC & $\mathrm{pRCC}$ & $\operatorname{chRCC}$ \\
\hline \multirow[t]{3}{*}{$\mathrm{RCC}$} & Total & 10 & 65.1 & 7 & 2 & 1 & 8 & 2 & 0 \\
\hline & Male & 7 & 68.6 & 6 & 1 & & 6 & 1 & 0 \\
\hline & Female & 3 & 57.0 & 1 & 1 & 1 & 2 & 1 & 0 \\
\hline \multirow[t]{3}{*}{ Control } & Total & 10 & 63.3 & & & & & & \\
\hline & Male & 6 & 64.8 & & & & & & \\
\hline & Female & 4 & 61.0 & & & & & & \\
\hline
\end{tabular}

RCC, renal cell carcinoma; ccRCC, clear cell RCC; pRCC, papillary RCC; chRCC, chromophobe RCC; TNM, Tumor-Node-Metastasis.

subsequently performed in triplicate for each sample using a SYBR ${ }^{\circledR}$ ExScript Real-time PCR kit (Takara Biotechnology Co., Ltd.). A $20 \mu \mathrm{l}$ reaction mixture was used, containing $2 \mu 1$ template DNA, $1 \mu 1$ primers, $10 \mu 1$ SYBR premix and $7 \mu \mathrm{dddH} 2 \mathrm{O}$. The primer sequences were as follows: $\beta$-actin forward, 5'-TGAAGGTGACAGCAGTCGGTT-3' and reverse, 5'-AGAAGTGGGGTGGCTTTTAGGA-3'; SALL4 forward, 5'-TTGCGACCACCCAAGTAT-3' and reverse, 5'-AACCCA CAGAACCAACCAC-3'. PCR was performed using a 7900HT Fast Real-Time PCR machine (Applied Biosystems; Thermo Fisher Scientific, Inc.) under the following conditions: $95^{\circ} \mathrm{C}$ for $30 \mathrm{sec}, 40$ cycles of $95^{\circ} \mathrm{C}$ for $5 \mathrm{sec}$ and $60^{\circ} \mathrm{C}$ for $30 \mathrm{sec}$. PCR results were quantified using the $-2^{\Delta \Delta \mathrm{Cq}}$ method (27).

ELISA. SALL4 in the serum was measured using a Human Sal-like protein 4(SALL4) ELISA kit (cat. no. EL020676HU; Miltenyi Biotec $\mathrm{GmbH}$ ) according to the manufacturer's instructions. The optical density (OD) at $450 \mathrm{~nm}$ was determined. A standard curve was established using OD450 as the $y$-axis and the concentration of a standard substance as the $\mathrm{x}$-axis; from this standard curve the level of protein was determined. Data are presented as the concentration of SALL4 $(\mathrm{ng} / \mathrm{ml})$ in samples.

Cell invasion assay. The invasive capability of OSRC-2 cells was determined by the Transwell assay $(8-\mu \mathrm{m}$ pore size; Corning, Inc.). OSRC-2 cells were harvested and seeded at $5 \times 10^{4}$ cells/well with serum-free DMEM into the upper chambers pre-coated with Matrigel $\left(37^{\circ} \mathrm{C}\right.$ for $\left.60 \mathrm{~min}\right)$, and the lower chambers contained DMEM with $10 \%$ FBS. Then, the Transwell assay was incubated for $24 \mathrm{~h}$ at $37^{\circ} \mathrm{C}$. Following incubation, the invasive cells attached to the lower surface of the membrane were fixed by $4 \%$ paraformaldehyde for $10 \mathrm{~min}$ at room temperature and stained with $1 \%$ toluidine blue for $10 \mathrm{~min}$ at room temperature. The number of cells penetrating across membrane was counted under a light microscope in ten random visual fields (magnification, x100).

MTT assay. Cell viability was assessed using MTT assay. After $24 \mathrm{~h}$ transfection with plasmids, SW839 or OSRC-2 cells were seeded at 1,000 per well in a 96-well plate. The cell proliferation assay was performed on days 1, 2, 3 and 4. MTT reagent $(5 \mathrm{mg} / \mathrm{ml})$ was added to each well and the plate was incubated for $2 \mathrm{~h}$ at $37^{\circ} \mathrm{C}$. The formazan crystals formed were solubilized in $100 \mu \mathrm{l}$ DMSO for $10 \mathrm{~min}$. Before the endpoint of incubation, the absorbance was measured at $450 \mathrm{~nm}$. Each sample was assayed in triplicate.

Statistical analysis. Statistical analyses were performed using SPSS 20 statistical software (SPSS, Inc.) and GraphPad Prism 7 (GraphPad Software, Inc.). Differences in mean values between two groups were analyzed by a two-tailed Student's t-test and the mean values of $>2$ groups were compared with one-way ANOVA with the Bonferroni post hoc test for multiple comparisons. Kaplan-Meier curves were calculated to determine if SALL4 expression was related to patient survival. Pearson's correlation was used to assess correlations between SALL4 gene expression and SALL4 copy number. $\mathrm{P}<0.05$ was considered to indicate a statistically significant difference.

\section{Results}

SALL4 expression is related to the survival of patients with $R C C$. The mRNA expression level of SALL4 and clinical information of 604 cases of ccRCC were obtained from TCGA using UCSC Xena (http://xena.ucsc.edu/). The patients with ccRCC were divided into SALL4-high or SALL4-low groups according to the median SALL4 mRNA expression level. The Kaplan-Meier curve was used to analyze if the expression level of SALL4 was related to the survival of patients with RCC. A significant difference $(\mathrm{P}<0.0001)$ was found between the two groups, where patients with lower SALL4 expression level had longer survival time compared with patients with higher SALL4 expression levels (Fig. 1A).

Similar analyses were carried out in patients with pRCC $(n=320)$ and chRCC $(n=89)$. A significant difference was found between SALL4 high and low expression groups in the pRCC cases $(\mathrm{P}=0.0006$; Fig. 1B). However, no significant difference was detected in chRCC in both groups $(\mathrm{P}=0.97$; Fig. 1C). Therefore, the present results suggested that patients with $\mathrm{pRCC}$ and low SALL4 mRNA expression have a longer survival time compared with patients with high SALL4 expression.

In addition, the prognostic relevance of sex was analyzed in $\mathrm{RCC}$, as RCC is reported to have a sex bias with a male to 

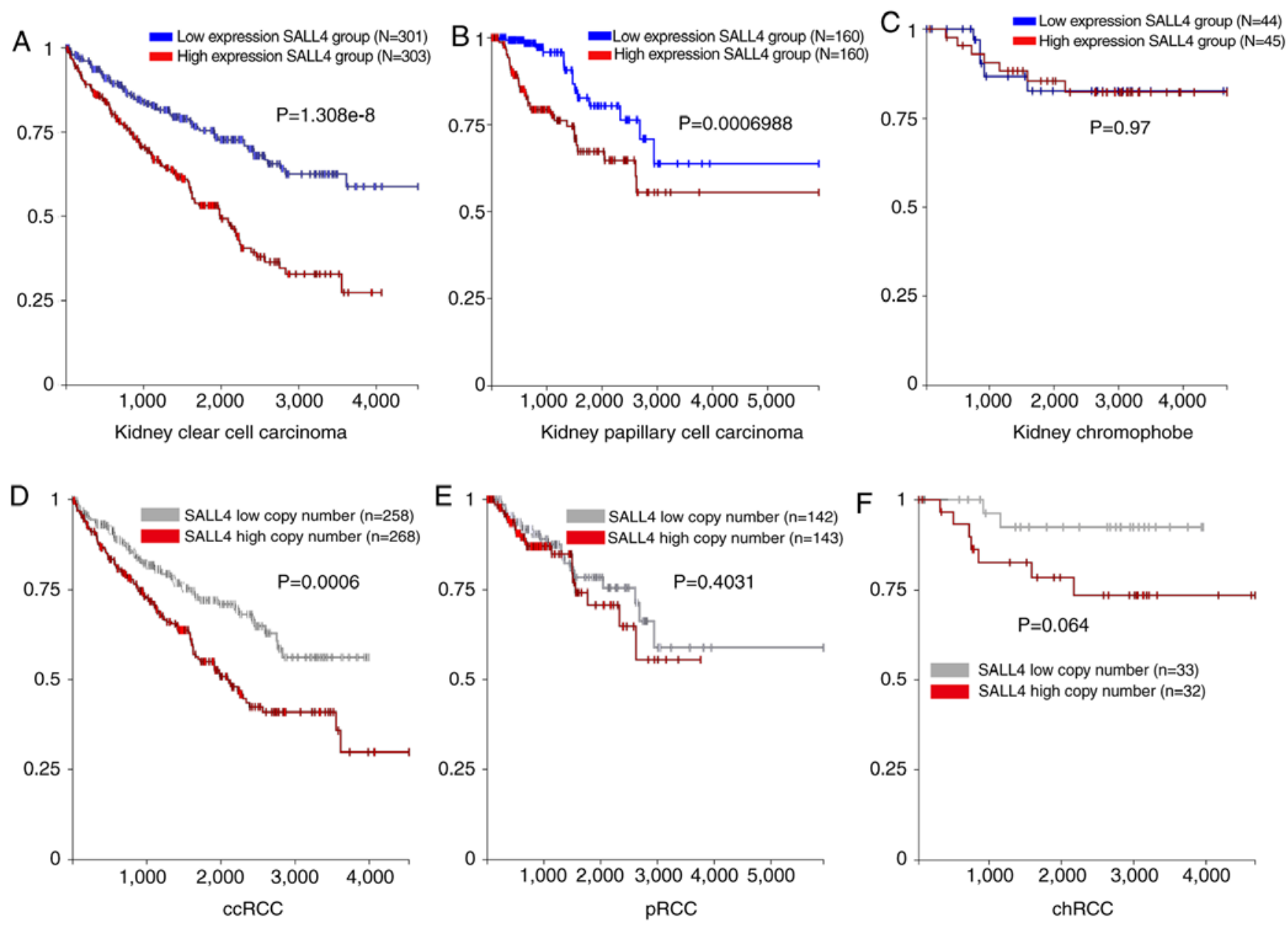

Figure 1. mRNA and copy number of SALL4, and SALL4 association with survival in ccRCC, pRCC and chRCC. Kaplan-Meier analysis of SALL4 mRNA expression level and OS in (A) ccRCC, (B) pRCC and (C) chRCC. Kaplan-Meier analysis of SALL4 copy number and OS in (D) ccRCC, (E) pRCC and (F) chRCC. OS, overall survival; SALL4, spalt like transcription factor 4; RCC, renal cell carcinoma; ccRCC, clear cell RCC; pRCC, papillary RCC; chRCC, chromophobe RCC.

female ratio of 2.3:1 (28). However, in the present study no significant difference was found in ccRCC, pRCC and chRCC in both SALL4 groups (data not shown). Therefore, while the incidence of RCC is higher in males compared with females, the outcome of RCC is the same in both sex.

SALL4 expression level in different stage and grades of RCC. The present study analyzed the expression level of SALL4 in different pathological TNM stages in the three RCCs groups. It was demonstrated that the expression level of SALL4 was higher in M1 compared with M0 ( $\mathrm{P}=0.0092)$. Moreover, as the pathology $\mathrm{T}$ and stage increased, the expression level of SALL4 was also significantly increased in ccRCC (Fig. 2A). Similar results were identified in patients with chRCC (Fig. 2C). However, the expression level of SALL4 showed no difference between different pathology $\mathrm{T}, \mathrm{M}$ and stage in pRCC (Fig. 2B). Collectively, the present results suggested that SALL4 promotes the progression of ccRCC and chRCC, but not pRCC.

SALL4 expression level between tumor and normal tissues in different tumors. To investigate the expression level of SALL4 in tumor and normal tissues, SALL4 expression level was assessed in different cancer types using FireBrowse software.
It was found that the expression level of SALL4 was higher in almost all cancer tissues compared with the normal tissues (Fig. S1A). For the three types of RCCs [KICH (chRCC), KIRC (ccRCC) and KIRP (pRCC)], it was demonstrated that the expression level of SALL4 was higher in the tumor tissue compared with the normal in ccRCC and chRCC. However, no difference was detected in pRCC (Fig. 3A). Therefore, the present results indicated that SALL4 may promote the progression of ccRCC and chRCC, but not pRCC.

Copy number of SALL4 in RCC. An increase in the copy number of SALL4 may be the putative mechanism underlying the high expression level of SALL4 in RCC. Thus, the present study analyzed the copy number of SALL4. The expression levels of the molecule and the clinical information of the 526 cases of ccRCC were obtained from TCGA using UCSC Xena (http://xena.ucsc.edu/). The patients with ccRCC were divided into SALL4-high or SALL4-low groups according to the median SALL4 copy number expression. The Kaplan-Meier curve was used to analyze the SALL4 copy number expression related to the survival of patients with RCC. A significant difference was found between the two groups $(\mathrm{P}=0.0006)$, similar to the results found for the mRNA expression level of SALL4 (Fig. 1D). However, 
A

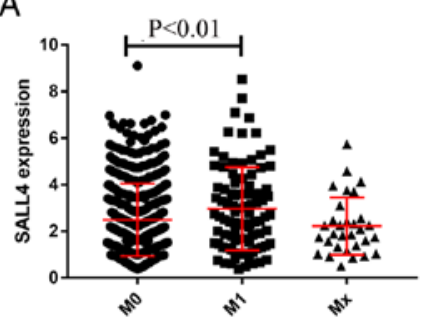

B

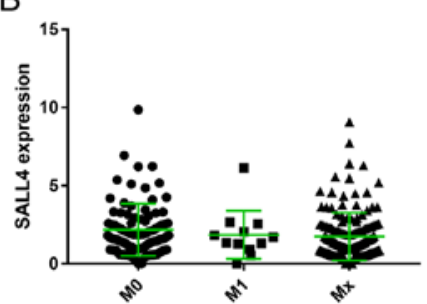

C

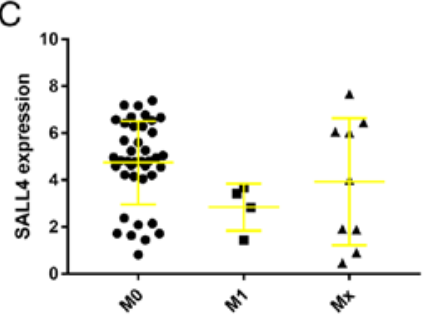

ccRCC

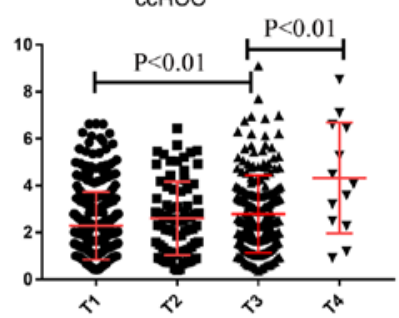

pRCC

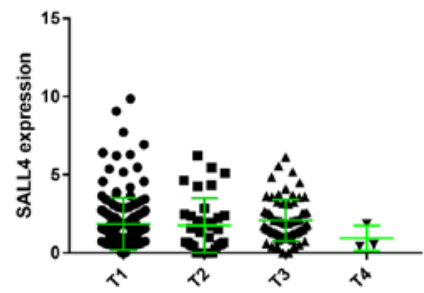

chRCC

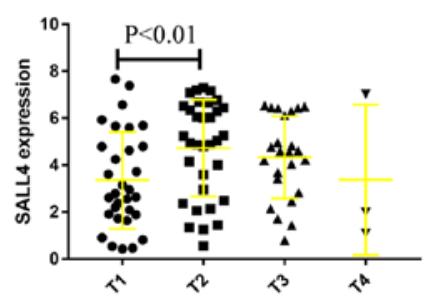

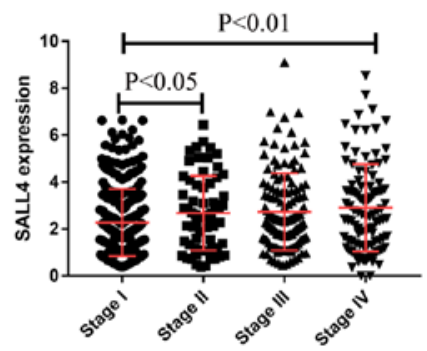
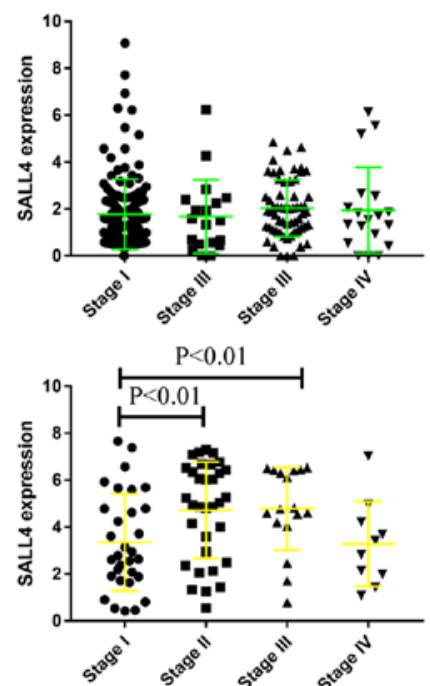

Figure 2. mRNA expression level of SALL4 and its association with pathology M, T and stage in ccRCC, pRCC and chRCC. (A) mRNA expression of SALL4 and its association with pathology M, T and stage in ccRCC. (B) mRNA expression of SALL4 and its association with pathology M, T and stage in pRCC. (C) mRNA expression of SALL4 and its association with pathology M, T and stage in chRCC. Data are presented as the mean \pm SD. SALL4, spalt like transcription factor 4; RCC, renal cell carcinoma; ccRCC, clear cell RCC; pRCC, papillary RCC; chRCC, chromophobe RCC.

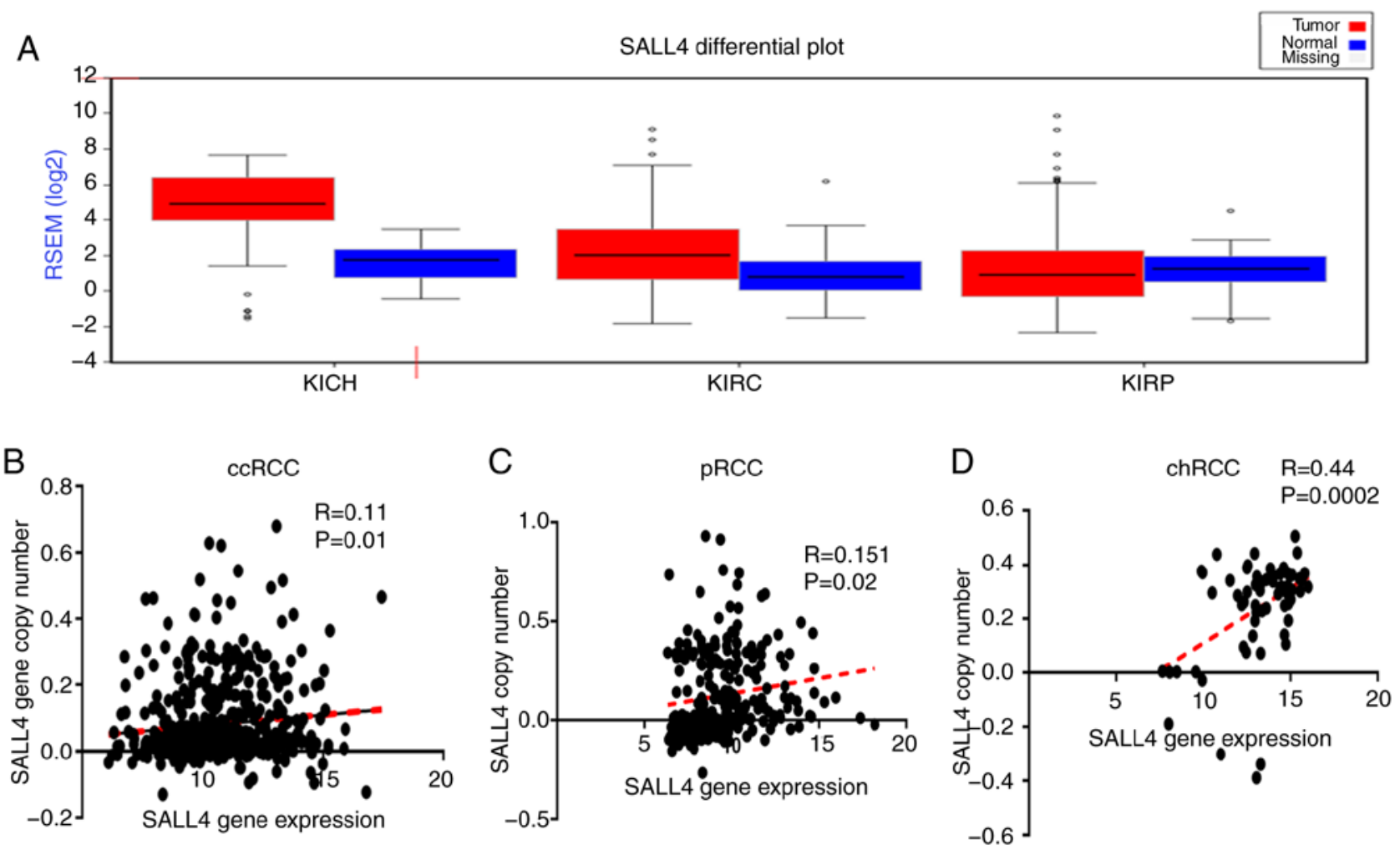

Figure 3. SALL4 expression and its relationship with SALL4 copy number in RCC. (A) SALL4 expression level in the three RCC [KICH (chRCC), KIRC (ccRCC) and KIRP (pRCC)] and healthy tissues. Correlation between SALL4 mRNA expression level and SALL4 copy number in (B) ccRCC, (C) pRCC and (D) chRCC. SALL4, spalt like transcription factor 4; RCC, renal cell carcinoma; ccRCC, clear cell RCC; pRCC, papillary RCC; chRCC, chromophobe RCC. 
Table II. List of top 20 genes that are related to SALL4 gene expression in ccRCC.

\begin{tabular}{lccc}
\hline Gene & $\operatorname{logFC}$ & P-value & adj. P-value \\
\hline AGR3 & -3.0309 & 0.0010 & 0.0561 \\
RAB25 & -2.7967 & 0.0180 & 0.1876 \\
CHGB & -2.6242 & 0.0053 & 0.1090 \\
SCNN1B & -2.6181 & 0.0333 & 0.2504 \\
APCDD1L & -2.4312 & 0.0122 & 0.1555 \\
MUC15 & -2.336 & 0.0488 & 0.3009 \\
HBG1 & -2.3191 & 0.0065 & 0.1181 \\
GPC5 & -2.2762 & 0.0090 & 0.1372 \\
CALML3 & -2.1989 & 0.0223 & 0.2081 \\
LY6H & -2.0310 & 0.0078 & 0.1277 \\
DCT & 1.9643 & 0.0000 & 0.0076 \\
SLC17A2 & 1.9728 & 0.0049 & 0.1054 \\
HIST1H2AJ & 2.0104 & 0.0033 & 0.0888 \\
MSLNL & 2.0332 & 0.0009 & 0.0526 \\
B4GALNT4 & 2.0702 & 0.0028 & 0.0816 \\
ADCY2 & 2.1198 & 0.0001 & 0.0019 \\
MOGAT1 & 2.1340 & 0.0018 & 0.0680 \\
ADAM18 & 2.3234 & 0.0138 & 0.1663 \\
TRIM72 & 2.3842 & 0.0071 & 0.1218 \\
MSLN & 2.5377 & 0.0003 & 0.0333 \\
\hline
\end{tabular}

FC, fold-change; $\log (\mathrm{FC})$, gene expression level; Adj. P-value, adjusted P-value; RCC, renal cell carcinoma; ccRCC, clear cell RCC.

no difference was observed in pRCC and chRCC (Fig. 1E and F). Furthermore, the correlation between SALL4 gene expression level and SALL4 copy number in ccRCC, pRCC and chRCC was found to have weak positive correlation between SALL4 gene expression and SALL4 copy number in $\operatorname{ccRCC}(\mathrm{R}=0.11 ; \mathrm{P}=0.01)$ and $\mathrm{pRCC}(\mathrm{R}=0.151 ; \mathrm{P}=0.02)$, and a moderate correlation in chRCC $(\mathrm{R}=0.44 ; \mathrm{P}=0.0002$; Fig. 3B-D).

Collectively, the present results suggested that the increase in SALL4 copy number may be one of the mechanisms for elevated SALL4 expression level in pRCC. However, the mechanism of high expression of SALL4 in ccRCC requires further investigation.

Enriched pathways and related genes correlates with SALL4 expression in RCC. Additionally, the present study examined the potential pathways, and identified the genes correlated to SALL4 using WebMeV (http://mev.tm4.org/\#/datasets/tcga). In total, 10 patients with high expression levels of SALL4 and 10 with low expression levels were analyzed. It was found that 19 pathways were significantly associated with SALL4 expression level in ccRCC (Fig. S1B). The top three pathways include translation, eukaryotic translation initiation and cap-dependent translation initiation, suggesting that SALL4 may be involved in the translation pathway.

Moreover, genes with expression levels that correlated with SALL4 expression levels in ccRCC were analyzed using
WebMeV (http://mev.tm4.org/\#/datasets/tcga). The expression levels of 2,674 genes were correlated with the expression level of SALL4 (data not shown). The top 20 genes whose expression levels were related to SALL4 are listed in Table II. Moreover, the present study did not identify any cancer stem cell genes such as NANOG, SOX2 and OCT4.

High expression levels of SALL4 in RCC samples and serum. To further examine the function of SALL4 in patients with RCC, the present study measured the protein expression levels of SALL4 in RCC tumors and healthy specimens using RT-qPCR and IHC. It was found that SALL4 protein expression level (yellow color) was higher in tumor samples compared with healthy specimens (Fig. 4A). Furthermore, the mRNA expression level of SALL4 was significantly higher in tumors compared with healthy specimens (Fig. 4B). In addition, expression level of SALL4 in the serum of 10 patients with RCC and 10 controls was measured. It was demonstrated that significantly higher serum SALL4 levels were presented in the patients with RCC compared with the controls. Furthermore, the mean serum SALL4 level was $4.03 \pm 0.61 \mathrm{ng} / \mathrm{ml}$ in the ccRCC group compared with $3.45 \pm 0.38 \mathrm{ng} / \mathrm{ml}$ in the control group $(\mathrm{P}<0.05$; Fig. 4C). The present study also measured the mRNA expression level in human renal tubular epithelial cells and the three RCC cell lines. It was found that SALL4 was significantly highly expressed in the three RCC cell lines (Fig. S2A).

SALL4 promotes cell viability and invasion in RCC cells. The present study performed lentivirus packaging of pLKO and sh-SALL4, which was then transduced into OSRC-2 and SW839 cells. RT-qPCR was used to examine the efficiency of transfection (Fig. S2B).

An invasion assay was performed in OSRC-2 cells, and the results indicated that knockdown of SALL4 decreased cell invasion compared with pLKO $(3.32 \pm 0.08$ vs. $1.05 \pm 0.11$; $\mathrm{P}<0.01$; Fig. 4D).

In addition, an MTT assay was used to assess the viability of OSRC-2 and SW839 cells. It was demonstrated that SALL4 knockdown decreased cell viability compared with the pLKO group $(\mathrm{P}=0.003$ in OSRC-2 and $\mathrm{P}=0.001$ in SW839 at day 3; Fig. 4E).

\section{Discussion}

In 2018, 403,000 new cases of kidney cancer were diagnosed worldwide, with a higher than $43 \%$ mortality rate in patients (29). A high incidence of small renal cancer is reported due to improved diagnosis, and $\sim 1 / 3$ of the patients with RCC develop metastatic lesions during the development of the disease (30). Moreover, nephrectomy (radial or partial) is the primary treatment for localized RCC; however, $>40 \%$ of patients with localized RCC exhibit a relapse or metastasis after surgery (31). Currently, the therapeutic targeting of the vascular endothelial growth factor using sunitinib, sorafenib, pazopanib, axitinib, tivozanib and cabozantinib, or of mTOR using everolimus, temsirolimus, and bevacizumab combined with interferon- $\alpha$ have been applied clinically to prolong survival in metastatic RCC (32). However, some patients are naturally resistant to these methods and most develop resistance (33), thus the treatment of RCC can be difficult. 
A

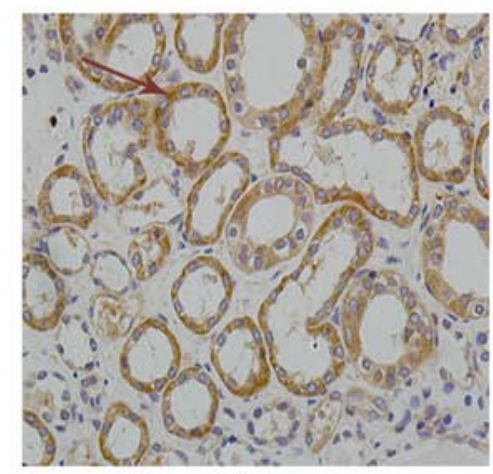

Normal kidney

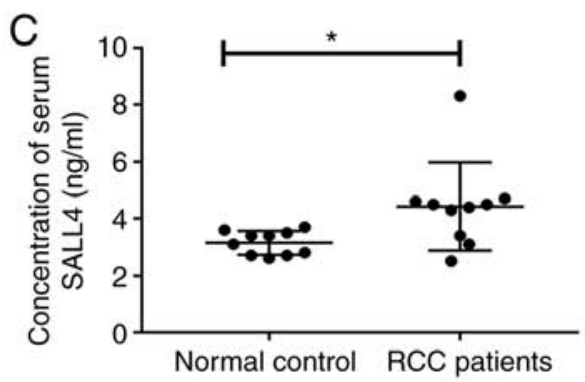

E

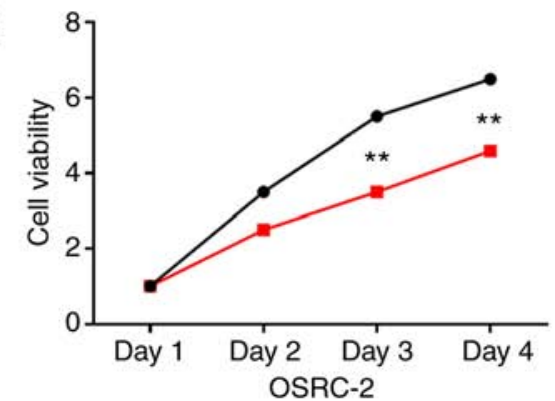

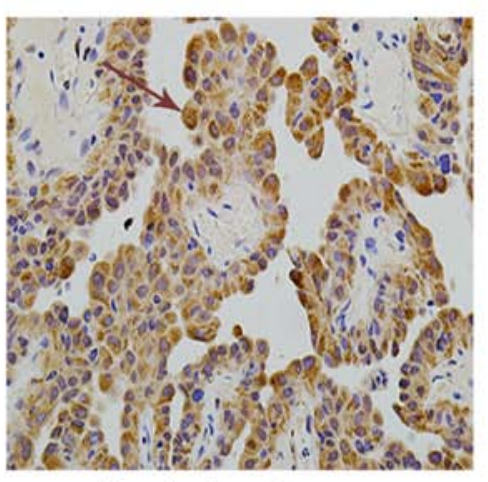

Renal cell carcinoma

D sh-pLKO

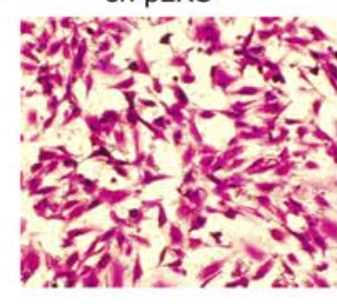

B

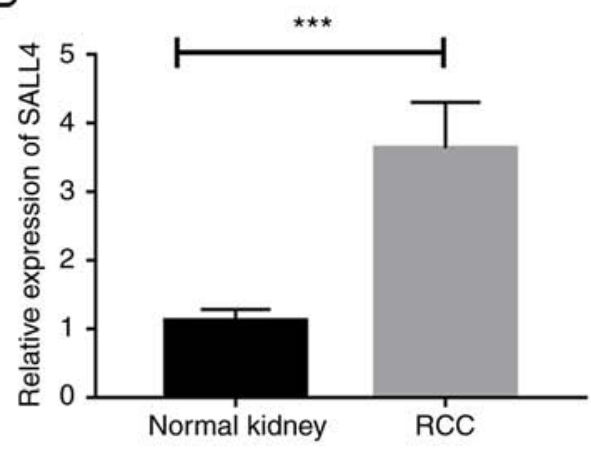

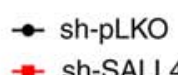

- sh-SALL4
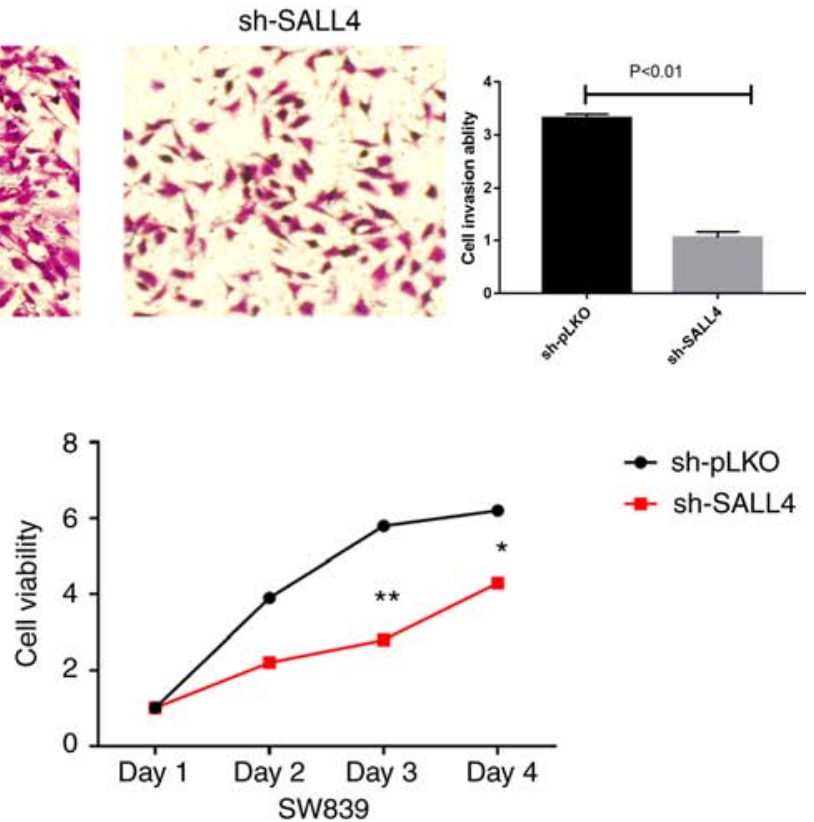

SW839

Figure 4. SALL4 function in RCC cells. (A) SALL4 protein expression level in tumor and healthy tissues. Magnification, $\mathrm{x} 400$. The yellow color indicated by the arrow represents the positive staining of SALL4. (B) SALL4 mRNA expression level in healthy kidney and RCC tissues. (C) Serum SALL4 level in patients with RCC and healthy controls. (D) Transwell assay results of cell invasion in OSRC-2 cells with knockdown of SALL4. Magnification, $x 400$. (E) SALL4 was knocked down in OSRC-2 and SW839 cells, and cell viability was examined by MTT. ${ }^{*} \mathrm{P}<0.05,{ }^{* *} \mathrm{P}<0.01,{ }^{* * *} \mathrm{P}<0.001$. SALL4, spalt like transcription factor 4 ; RCC, renal cell carcinoma; sh, short hairpin RNA.

Therefore, further investigations on the molecular mechanisms underlying the metastasis or progression of ccRCC, and into new novel targets are urgently required. Currently, there is no reliable biomarker for RCC, unlike prostate-specific antigen for prostate cancer (34). Thus, identifying novel and reliable prognostic biomarkers for patients with RCC is important to predict patient outcomes and facilitate effective clinical management.

SALL4, a member of the spalt-like gene family, is a critical stem cell factor. SALL4 is a zinc finger transcription factor that is enriched in the embryonic cell. Moreover, SALL4 plays a major role in the self-renewal capability, while its expression is silenced in the mature adult (35). A previous study showed that SALL4 is crucial for maintaining the stemness properties of embryonic stem cells (36). Another previous study demonstrated that SALL4 controls the stemness properties of embryonic stem cells at both the transcriptional and epigenetic levels via direct or indirect interaction with Nanog and OCT4 (20). However, it has also been showed that SALL4 is re-expressed in various cancer types (11-16) and was first recognized as an oncogene in leukemia $(17,37)$. Yakaboski et al (38) found that in SALL4-positive HCC, the expression of certain progenitor-like genes is high. Other studies have also confirmed a critical role of SALL4 in cell survival and tumorigenicity by knocking down SALL4 (39). Zhang et al (20) demonstrated that the overexpression of SALL4 in gastric cancer cells promotes cell stemness by increasing the expression levels of other cancer stem cell markers such as CD133, SOX2, Bmi-1 and Lin28. Furthermore, SALL4 has been identified as a core factor in the SALL4/Nanog/Oct4 network (9). Moreover, as a transcription factor, SALL4 can activate Oct4 and interact with Nanog $(9,40)$. Therefore, as a cancer stem cell marker, SALL4 plays a major role in cancer formation. However, the present study searched the top 20 genes related to SALL4, and identified genes that may be attributed to the varied SALL4 pathway in different cancer types.

The present study investigated the clinical value of SALL4 in RCC and demonstrated that the expression level of SALL4 was associated with survival, stage and pathology $\mathrm{T}$, thus 
indicating that SALL4 is a poor prognostic factor for a poor outcome in RCC. In addition to being a biomarker for RCC diagnosis, SALL4 may also be a potential therapeutic target. A previous study found that the inhibition of SALL4 expression by siRNA reduces cell survival, and impairs the migration and invasion of indistinct cancer cells in vitro (20). Moreover, targeting SALL4 using PTEN (23) or entinostat (24) may have therapeutic efficacy in both acute myeloid leukemia and lung cancer. Thus, it can be hypothesized that targeting SALL4 using miRNA, PTEN or entinostat may have therapeutic efficacy in RCC.

The mechanism underlying the high expression level of SALL4 and its downstream genes in RCC is not fully understood. The present results suggested that the copy number of SALL4 was increased in ccRCC. Additionally, the copy number of SALL4 was positively associated with the survival curve. Moreover, a positive correlation was established between SALL4 mRNA and copy number in ccRCC, pRCC and chRCC, indicating that an increased copy number may be the mechanism underlying the high expression of SALL4 in RCC. The enriched pathways analysis results identified several genes and pathways that may be associated with SALL4; however, the cancer stem cell marker gene and related pathway were not deduced as SALL4 is involved in multiple pathways promoting cancer progression. Nevertheless, the downstream genes and the mechanism via which SALL4 promotes RCC progression requires further investigation. The present study used specimens and serum from patients with RCC to identify the high expression level of SALL4. Furthermore, using the MTT and invasion assays, it was found that SALL4 promotes cell viability and invasion in RCC cells. To the best of our knowledge, this is the first study to investigate the expression levels of SALL4 in the serum and tumor specimens in RCC patients. Moreover, targeting this newly identified SALL4 signaling pathway may facilitate the development of novel therapies to treatment RCC and improved survival rates. However, there were limitations to the present study. Firstly, only three most common types of RCC were analyzed due to TCGA data limitation. Secondly, the downstream pathways or factors of SALL4 were not identified. In addition, the patients with RCC only had 1 year follow-up data, thus the survival curve is not valuable. Furthermore, the cause of increased SALL4 expression levels in patients with RCC is still unknown.

In conclusion, the present results suggested that SALL4 may be a sensitive and specific cancer biomarker in ccRCC and pRCC. Thus, targeting SALL4 may improve RCC therapy and prolong the survival of patients with ccRCC and pRCC.

\section{Acknowledgements}

The results of this study are partially based on the data generated by the TCGA Research Network (https:/www.cancer. gov/tcga).

\section{Funding}

This study was funded by the National Natural Science Foundation of China (grant no. 81802518).

\section{Availability of data and materials}

The datasets used and/or analyzed during the current study are available from the corresponding author on reasonable request.

\section{Authors' contributions}

JC, CG, and PW were involved in creating/designing the study, data collection and data analysis. JC and CG wrote the manuscript. JZ, GW and XY were involved in project development and data collection. All authors have read and approved the final version of the manuscript.

\section{Ethics approval and consent to participate}

The present study analyzed the raw data that is published in The Cancer Genome Atlas. The current study was performed according to the protocol approved by the Ethics Committee of Shanghai Tenth People's Hospital, Tongji University School of Medicine. Written informed consent for participation was obtained from each patient.

\section{Patient consent for publication}

Not applicable.

\section{Competing interest}

The authors declare that they have no competing interests.

\section{References}

1. Rini BI, Campbell SC and Escudier B: Renal cell carcinoma. Lancet 373: 1119-1132, 2009.

2. King SC, Pollack LA, Li J, King JB and Master VA: Continued increase in incidence of renal cell carcinoma, especially in young patients and high grade disease: United States 2001 to 2010. J Urol 191: 1665-1670, 2014.

3. Ljungberg B, Bensalah K, Canfield S, Dabestani S, Hofmann F, Hora M, Kuczyk MA, Lam T, Marconi L, Merseburger AS, et al: EAU guidelines on renal cell carcinoma: 2014 update. Eur Urol 67: 913-924, 2015.

4. Linehan WM and Ricketts CJ: Decade in review-kidney cancer: Discoveries, therapies and opportunities. Nat Rev Urol 11: 614-616, 2014.

5. Choueiri TK and Motzer RJ: Systemic therapy for metastatic renal-cell carcinoma. N Engl J Med 376: 354-366, 2017.

6. Al-Baradie R, Yamada K, St Hilaire C, Chan WM, Andrews C, McIntosh N, Nakano M, Martonyi EJ, Raymond WR, Okumura $\mathrm{S}$, et al: Duane radial ray syndrome (Okihiro syndrome) maps to $20 \mathrm{q} 13$ and results from mutations in SALL4, a new member of the SAL family. Am J Hum Genet 71: 1195-1199, 2002.

7. Kohlhase J, Schuh R, Dowe G, Kühnlein RP, Jäckle H, Schroeder B, Schulz-Schaeffer W, Kretzschmar HA, Köhler A, Müller U, et al: Isolation, characterization, and organ-specific expression of two novel human zinc finger genes related to the Drosophila gene spalt. Genomics 38: 291-298, 1996.

8. Tatetsu H, Kong NR, Chong G, Amabile G, Tenen DG and Chai L: SALL4, the missing link between stem cells, development and cancer. Gene 584: 111-119, 2016.

9. Yang J, Gao C, Chai L and Ma Y: A novel SALL4/OCT4 transcriptional feedback network for pluripotency of embryonic stem cells. PLoS One 5: e10766, 2010.

10. Rao S, Zhen S, Roumiantsev S, McDonald LT, Yuan GC and Orkin SH: Differential roles of Sall4 isoforms in embryonic stem cell pluripotency. Mol Cell Biol 30: 5364-5380, 2010. 
11. Cao D, Li J, Guo CC, Allan RW and Humphrey PA: SALL4 is a novel diagnostic marker for testicular germ cell tumors. Am J Surg Pathol 33: 1065-1077, 2009.

12. Zhang L, Yan Y, Jiang Y, Cui Y, Zou Y, Qian J, Luo C, Lu Y and Wu X: The expression of SALL4 in patients with gliomas: High level of SALL4 expression is correlated with poor outcome. J Neurooncol 121: 261-268, 2015.

13. Cao D, Guo S, Allan RW, Molberg KH and Peng Y: SALL4 is a novel sensitive and specific marker of ovarian primitive germ cell tumors and is particularly useful in distinguishing yolk sac tumor from clear cell carcinoma. Am J Surg Pathol 33: 894-904, 2009.

14. Mei K, Liu A, Allan RW, Wang P, Lane Z, Abel TW, Wei L, Cheng H, Guo S, Peng Y, et al: Diagnostic utility of SALL4 in primary germ cell tumors of the central nervous system: A study of 77 cases. Mod Pathol 22: 1628-1636, 2009.

15. Li A, Jiao Y, Yong KJ, Wang F, Gao C, Yan B, Srivastava S, Lim GS, Tang P, Yang H, et al: SALL4 is a new target in endometrial cancer. Oncogene 34: 63-72, 2015.

16. Ushiku T, Shinozaki A, Shibahara J, Iwasaki Y, Tateishi Y, Funata $\mathrm{N}$ and Fukayama M: SALL4 represents fetal gut differentiation of gastric cancer, and is diagnostically useful in distinguishing hepatoid gastric carcinoma from hepatocellular carcinoma. Am J Surg Pathol 34: 533-540, 2010

17. Ma Y, Cui W, Yang J, Qu J, Di C, Amin HM, Lai R, Ritz J, Krause DS and Chai L: SALL4, a novel oncogene, is constitutively expressed in human acute myeloid leukemia (AML) and induces AML in transgenic mice. Blood 108: 2726-2735, 2006.

18. Wu M, Yang F, Ren Z, Jiang Y, Ma Y, Chen Y and Dai W: Identification of the nuclear localization signal of SALL4B, a stem cell transcription factor. Cell Cycle 13: 1456-1462, 2014.

19. Jeong HW, Cui W, Yang Y, Lu J, He J, Li A, Song D, Guo Y, Liu BH and Chai L: SALL4, a stem cell factor, affects the side population by regulation of the ATP-binding cassette drug transport genes. PLoS One 6: e18372, 2011.

20. Zhang X, Yuan X, Zhu W, Qian $\mathrm{H}$ and $\mathrm{Xu}$ W: SALL4: An emerging cancer biomarker and target. Cancer Lett 357: 55-62, 2015.

21. Yong KJ, Chai L and Tenen DG: Oncofetal gene SALL4 in aggressive hepatocellular carcinoma. N Engl J Med 369: 1171-1172, 2013

22. Zhang L, Xu Z, Xu X, Zhang B, Wu H, Wang M, Zhang X, Yang T, Cai J, Yan Y, et al: SALL4, a novel marker for human gastric carcinogenesis and metastasis. Oncogene 33: 5491-5500, 2014.

23. Gao C, Dimitrov T, Yong KJ, Tatetsu H, Jeong HW, Luo HR, Bradner JE, Tenen DG and Chai L: Targeting transcription factor SALL4 in acute myeloid leukemia by interrupting its interaction with an epigenetic complex. Blood 121: 1413-1421, 2013.

24. Yong KJ, Li A, Ou WB, Hong CK, Zhao W, Wang F, Tatetsu H, Yan B, Qi L, Fletcher JA, et al: Targeting SALL4 by entinostat in lung cancer. Oncotarget 7: 75425-75440, 2016.

25. May M, Surcel C, Capitanio U, Dell'Oglio P, Klatte T, Shariat S, Ecke T, Wolff I, Vergho D, Wagener N, et al: Prognostic and discriminative power of the 7th TNM classification for patients with surgically treated papillary renal cell carcinoma: Results of a multi-institutional validation study (CORONA subtype project). Scand J Urol 51: 269-276, 2017.
26. Zhai W, Sun Y, Guo C, Hu G, Wang M, Zheng J, Lin W, Huang Q, $\mathrm{Li} \mathrm{G}$, Zheng J and Chang C: LncRNA-SARCC suppresses renal cell carcinoma (RCC) progression via altering the androgen receptor(AR)/miRNA-143-3p signals. Cell Death Differ 24: 1502-1517, 2017.

27. Livak KJ and Schmittgen TD: Analysis of relative gene expression data using real-time quantitative PCR and the 2(-Delta Delta C(T)) method. Methods 25: 402-408, 2001.

28. He D, Li L, Zhu G, Liang L, Guan Z, Chang L, Chen Y, Yeh S and Chang C: ASC-J9 suppresses renal cell carcinoma progression by targeting an androgen receptor-dependent HIF $2 \alpha /$ VEGF signaling pathway. Cancer Res 74: 4420-4430, 2014.

29. Bray F, Ferlay J, Soerjomataram I, Siegel RL, Torre LA and Jemal A: Global cancer statistics 2018: GLOBOCAN estimates of incidence and mortality worldwide for 36 cancers in 185 countries. CA Cancer J Clin 68: 394-424, 2018.

30. Petejova N and Martinek A: Renal cell carcinoma: Review of etiology, pathophysiology and risk factors. Biomed Pap Med Fac Univ Palacky Olomouc Czech Repub 160: 183-194, 2016.

31. Ravaud A, Motzer RJ, Pandha HS, George DJ, Pantuck AJ, Patel A, Chang YH, Escudier B, Donskov F, Magheli A, et al: Adjuvant Sunitinib in High-Risk Renal-Cell Carcinoma after Nephrectomy. N Engl J Med 375: 2246-2254, 2016.

32. Ljungberg B, Albiges L, Abu-Ghanem Y, Bensalah K, Dabestani S, Fernández-Pello S, Giles RH, Hofmann F, Hora M, Kuczyk MA, et al: European association of urology guidelines on renal cell carcinoma: The 2019 update. Eur Urol 75: 799-810, 2019.

33. Rini BI and Atkins MB: Resistance to targeted therapy in renal-cell carcinoma. Lancet Oncol 10: 992-1000, 2009.

34. Pezaro C, Woo HH and Davis ID: Prostate cancer: Measuring PSA. Intern Med J 44: 433-440, 2014.

35. Yang J: SALL4 as a transcriptional and epigenetic regulator in normal and leukemic hematopoiesis. Biomark Res 6: 1, 2018.

36. Yang L, Liu L, Gao H, Pinnamaneni JP, Sanagasetti D, Singh VP, Wang K, Mathison M, Zhang Q, Chen F, et al: The stem cell factor SALL4 is an essential transcriptional regulator in mixed lineage leukemia-rearranged leukemogenesis. J Hematol Oncol 10: 159, 2017.

37. Farawela HM, Zawam HM, Al-Wakeel HA, El-Nagdy MH, El-Refaey FA and Abdel-Rahman HA: Expression pattern and prognostic implication of SALL4 gene in myeloid leukemias: A case-control study. Scand J Clin Lab Invest 79: 65-70, 2019.

38. Yakaboski E, Jares A and Ma Y: Stem cell gene SALL4 in aggressive hepatocellular carcinoma: A cancer stem cell-specific target? Hepatology 60: 419-421, 2014

39. He J, Zhou M, Chen X, Yue D, Yang L, Qin G, Zhang Z, Gao Q, Wang D, Zhang C, et al: Inhibition of SALL4 reduces tumorigenicity involving epithelial-mesenchymal transition via Wnt/ $\beta$-catenin pathway in esophageal squamous cell carcinoma. J Exp Clin Cancer Res 35: 98, 2016.

40. Wang J, Rao S, Chu J, Shen X, Levasseur DN, Theunissen TW and Orkin SH: A protein interaction network for pluripotency of embryonic stem cells. Nature 444: 364-368, 2006.

This work is licensed under a Creative Commons Attribution-NonCommercial-NoDerivatives 4.0 International (CC BY-NC-ND 4.0) License. 